\title{
Why minds cannot be received, but are created by brains
}

\section{WŁODZISŁAW DUCH}

Department of Informatics, Faculty of Physics, Astronomy and Informatics, and Neurocognitive Laboratory, Center for Interdisciplinary Modern Technologies, Nicolaus Copernicus University, Toruń, Poland wduch@is.umk.pl

\begin{abstract}
There is no controversy in psychology or brain sciences that brains create mind and consciousness. Doubts and opinions to the contrary are quite frequently expressed in non-scientific publications. In particular the idea that conscious mind is received, rather than created by the brain, is quite often used against "materialistic" understanding of consciousness. I summarize here arguments against such position, show that neuroscience gives coherent view of mind and consciousness, and that this view is intrinsically non-materialistic.
\end{abstract}

Keywords: brain; mind; consciousness; soul; neuroimaging; artificial intelligence; personal identity; dynamical forms.

\section{Introduction}

In recent decades molecular biology, neuroscience and cognitive sciences have made great progress in unravelling the mysteries of life, understanding how organisms function and control their behavior. Already at the end of the 19th century dualistic explanation placing soul that animates the body as the center of cognition has been abandoned in scientific literature. 
In philosophical literature the psycho-physical problem is still a matter of discussion and speculation, but cognitive sciences, including psychology, neuroscience, artificial intelligence and the science of consciousness (Dehaene 2014) have verified many hypothesis unraveling the mechanisms of mental life.

Understanding how embodiment and the ability to act in the world has facilitated specific forms of cognition, how dynamic interactions with environment shapes cognition, is fairly recent. Emergent self-organized complex systems arise from interactions of simple elements, leading to qualitatively new forms of organization and interaction. Cognitive systems do not receive passively information from their environment but actively enact it, in this way transforming their environment and generating intrinsic meaning. Systems of sufficient complexity create models of their environment and the self as agent, increasing the chances of survival by predicting the results of their own actions.

Many animals have brains that are sufficiently complex to allow for imagery and representation of the self. Human brains, with greatly expanded association cortex, are capable of creating rich mental models of the world, self and other minds, understanding intentions of humans and animals. Awareness based on perception, attention and reaction to environmental events is omnipresent in animal kingdom and has already been implemented in robots (Edelman 2006, 2007; Cruse H. \& Schilling M. 2015). Social animals need sophisticated communication. Human brains have sufficient power to develop the ability to comment on the state of their internal models (perceptions, associations, thoughts) using rich symbolic language. Without such language internal narration describing the stream of mental events is quite poor. The behavioral reactions of animals reflect their mental processes, such as reading intentions and anticipation of actions of other animals, planning coordinated actions during hunting. Motor activity of dogs in sleep seems to be a result of dreams. In the brains of animals neuronal space available for association and self-reflection is limited, but many mammals and even birds have complex social life, and primates develop sense of justice and unique cultures (de Waal 1996, 2005). 
Scientific ideas are difficult to comprehend without proper education. Traditional explanation of mind as "a ghost in the machine" is much simpler, intuitively compelling and deeply entrenched in our culture. There are good evolutionary reasons for this situation (Bering 2006). They do not give any understanding of specific issues, cannot be verified, but for most people are satisfactory. Mental processes are qualitatively quite different from physical processes, but qualitative differences are frequently seen in science as different facets of the same phenomenon. Two explosive gases mixed together make water with completely different properties. Images on game console screen, sounds of personal assistant produced by smartphones, are qualitatively different than currents or voltages flowing through the integrated circuits, but images and sounds that carry rich information are just another interpretation of the activity of electronic systems. The same information may also take form of electromagnetic waves, passed from the satellite. Expression of this information needs physical system that can change it into signals stimulating our senses (or directly our brains). Mental states are qualitatively different than neural activity of the brain, but they are just another interpretation of neurodynamics. Expression of these states requires body movements, gestures, speech.

In this paper I will summarize arguments that show why mind cannot be received from external sources. Next I shall discuss relations between matter, form and information, introducing the concept of dynamical form. A short discussion on relation of our current knowledge to traditional ideas closes this paper.

\section{Baggage of the antiquity}

The need to understand the world, explain observations and predict what will happen, led to development of language and creation of mental models. Concepts were gradually introduced, from simple symbol designating objects and actions, to metaphors helping to explain some phenomena and understand intentions. Concepts that initially referred to concrete objects, for example the Greek word anemos, that initially meant wind or breath, 
started to signify an abstract idea of animation, and were later reified into an abstract concept of anima, or the essence of life. Aristotle in De anima (2016) writes that psyche comes from respiration and refrigeration (katapsyxis in Greek). The history of such concepts is long, it may be linked to earlier ideas developed by Egyptian, Sumerian, Indian and other cultures. They had a clear explanatory function. Matter does not move by itself, there must be a mover inside. In Aristotle time the concept of the soul was well established. In his treatise De anima he mentions earlier ideas of Democritus, Pythagoras, Anaxagoras, Plato and several other early Greek philosophers. For him "the soul is in some sense the principle of animal life", so the study of the soul "must fall within the science of nature". Many ideas discussed in ancient works seem rather strange to scientists today. Some ideas that were discussed in Aristotle times seemed unreasonable to him, for example the soul as a self-moving number. His conclusion is that souls are needed to distinguish objects that are capable of living, performing their natural functions (seeds, fruits, animal bodies), from those that are unable to do it. Moreover, since various "powers" or functions of living things are different it makes sense to categorize souls according to at least three powers: nutritive (vegetative), sensitive and rational (Aristotle 2016).

Aristotle saw the need for explanation of what makes the living thing alive, what unifies the elements of the body, and allows them to function. There must be something in addition to the matter that animates it, and this animating principle was at his time called soul. Is it just the special form of the matter, or is it something in addition to the matter? The animal body changes from birth to death, but life force is present. The simplest explanation is that souls of animals and humans continue to animate the body, but are inseparable from the body. Aristotle rejected the idea of souls independent of the body opting for a holistic idea that is known as hylomorphism. Living things are composed of matter, but it has a complex form. They are alive as long as this form is not significantly damaged. Specific form makes activity of things, animals and people possible, so form is their soul. 
Hylomorphism has been embraced by theologians, especially in Thomas Aquinas tradition. The idea of form as the essence of life can be extended to dynamical forms, and reconciled with neuroscience's view of the mind. Current research on consciousness has reviewed many speculations related to the mind-body relation, including quantum processes, panpsychism, nonreductive naturalistic dualism and many others. The idea that brain matter needs some external guidance to explain mental processes has not yet been universally rejected in philosophy, although the only progress in understanding minds has been done in cognitive sciences. There seems to be a general agreement that some brain functions, such as perception, attention, sensorimotor actions, are brain functions that are explained in details by neuroscience. We understand these functions and have implemented some of them in robots (Edelman 2006, 2007). The controversy has focused on conscious mental states and creation of subjective experience. Here the idea that conscious mind is received by the brains is still discussed. For example, John Eccles and Karl Popper wrote: “...we have to recognize that we are spiritual beings with souls existing in a spiritual world as well as material beings with bodies and brains existing in a material world" (Eccles \& Popper 1989, 241). To explain how souls interact with brains Eccles has proposed a strange quantum hypothesis of "psychons". Search for the soul at the quantum level continues (Beauregard \& O’Leary 2007). Such approaches do not try to explain facts known from cognitive psychology or neurosciences. Internet search for "brain as a receiver of consciousness" shows thousands of web pages that make such claims, although they are rarely published in scientific journals (Sleutjes et al. 2014).

Stories invoked to sustain dualistic ideas are connected to the near-death experiences (NDE). Exiting body, seeing it from above, moving to other places during the cardiac arrest period and other such reports have anecdotal status. Attempts to verify that the out-of-body experiences reported by people that were resuscitated are real led to a large scale AWARE (AWAreness during REsuscitation) project, lasting 4 years. 15 medical centers were involcd and over 2000 cases have been examined. Review of the results (Parnia 2014) has not confirmed reality of these experiences, not a single subject has read 
the writings that could be seen only from above in the emergency room. Claims that there was no brain activity or EEG signal was flat are not based on proper measurements. The lack of signals from a single central EEG electrode is not an indication that there is no activity in the whole brain, especially in temporal lobes and hippocampus that may store auditory information. It is impossible to use any neuroimaging technique under resuscitation conditions, as the setting up of equipment takes a long time. Residual brain activity, although not conscious, may allow for storing some information that is later re-lived and attributed to the time of resuscitation. Illusions of being outside own body, having additional limbs or virtual body of different sizes, being invisible, can be artificially induced. It is a fascinating area of research in neuroscience (see for example work done in H. Ehrsson laboratory, http://www.ehrssonlab.se).

\section{Consciousness as a function of the brain}

Claims that "mind" or "consciousness" is received by the brain from some external source - called here mind or soul - have not defined precisely which functions of the mind or consciousness are received. The vegetative or responsive faculties are rather noncontroversial, there is nothing to receive. The rational soul has been equated by Aquinas with mind and intellect:

...the principle of intellectual operation which we call the soul ...We must conclude, therefore, that the human soul, which is called the intellect or the mind, is something incorporeal and subsistent. (Summa Theologica I, q. 75, a. 2)

It does not make sense to look for spiritual souls, intellect or mind as received, not created by the brain. Some arguments that are against such dualistic understanding are listed below. I shall use the word mind for mental processes in hylomorphic sense, and soul in dualistic sense. I shall use the concept of Self to signify all mental processes that I may be conscious of linked to the ideas about me and my relations with the world. 


\subsection{Complexity of brains}

Everything in biology at molecular and cellular level is incredibly complex. These complex processes in worms or insects produce relatively simple functions allowing for feeding and reproduction. Ticks will wait for months on grass or leaves for their victim. Their behavior is fairly simple but is supported by great complexity of insect organism, genetic, biochemical and neural processes. Human brains have the most complex structures in the known Universe, enabling complex mental states (Bassett and Gazzaniga 2011). Human behavior results from changes in our biological organism, formation of trillion connections between almost 100 billion neurons and functional synchronization of all cells in the organism. Precise control of brains, needed for conscious experience, would require even higher complexity of the controlling entity. Moreover, since embodiment and interaction with the world is necessary to form new connections in the brain the information flow between the brain and the soul would have to be enormous. Our thinking and experience is directed towards physical environment and social relations. Souls in their own realm would have to learn all about this world to be able to transmit anything relevant to our activity and experience.

Human organism contains about 50 trillion cells, with about two meters of DNA chain in each cell. The total length of DNA in our body is about 100 billion $\mathrm{km}$ or 666 larger than the distance to the Sun. Bacteria, viruses, fungi, archaea and other microbes outnumber the body cells. In the central nervous system 100 billion neurons are connected by a quadrillion of synapses. A few million of new connections between neurons are formed in each second during the first years of life, linking different brain areas. Their growth is controlled by neurotrophic factors but sensory stimulation (including motor proprioception) is need to facilitate correct structure and function. Each cell contains 10 billion protein molecules, and more than 500.000 types of proteins are known. Organism is not a fixed structure, it is an evolving process, and it needs to interact with incredibly complex ecosystem, from bacteria and other microorganisms to plants and animals. Some cells die after only 4 days (for example gut cells), but other cells (neurons) may live 
for 100 years, helping to preserve long-term memory and contributing to the feeling of personal identity.

Mental and biological processes are supported by huge complexity of brains and bodies, substrates in which complex minds and bodies may arise, if conditions are right. Evolutionary biology provides a framework in which environment through genetic selection creates a specific form of the body and of the mind, including representation of the Self. The body with the brain that has a great potential or development is born, but the Self is formed over the years, starting from simple inborn reflexes, to development of perception, language, the theory of mind and the ability for moral reasoning. I could have not been born at another time in history or in another country. A child born in China, even from the same parents, would develop quite different Self. Some character traits that are due to the genetic factors could be similar, but the view of the world, value system, the feeling of personal identity in relation to other people would reflect local culture, and so personal identity would be different.

Individual identity is closely related to experience and memory encoded in the substrate of brain matter. Only a very small percentage of brain processes are consciously accessible and may be perceived at the mental level. Regulation of life processes in such complex organism is done by homeostatic mechanisms that are not accessible to the conscious Self. The Self is not able to control cell growth, homeostasis or neural processes required to make the simplest movement. Neuronal activity changes very rapidly synchronizing multiple cortical and subcortical brain areas. The brain has learned to label (assign words) activation that happens repeatedly, and these labels are used for communication, pointing to the events and objects in the world, or to internal states (imagery, thoughts). Inner perception includes emotional value associated with mental states. According to the "radical plasticity" hypothesis of Cleeremans (2011) brains learn to be conscious, predicting their own internal activity as well as the consequences of external actions on the world and on other agents.

Comparing to complexity of brain processes mental processes are rather simple. Behind each conscious decision, each thought or gesture, 
large synchronized activity of the most complex machinery imaginable is hidden. Mind-brain relations, or subjective-objective perspectives on human behavior, may be summarized in a metaphor: Mind is a shadow of neurodynamics (Duch, 2012). Neural activity may be measured using various techniques: EEG, MEG, NIRS, PET, fMRI, while mental events are represented by qualities recognized in the inner experience. Sometimes this experience is hard to describe, for example in the unusual brains states (in dreams, illusions, psychoactive drugs, or direct brain stimulation), or in situations when the brain has not yet learned how to interpret new experiences (Schwitzgabel, 2011).

Brains support minds because their structure has enormous complexity. Chips in computers and smartphones contain billions of elements and therefore enable many complex functions. They are "dead" when the energy source (battery) is disconnected and spring to life when energy is supplied. Electronic devices need power supply to function, but their "soul" is in their whole organization, connection structure, memorized programs and data. Brains also need energy to function, but the essence of their identity is in their complex structure that is learned from individual experiences (Bassett and Gazzaniga 2011). Learning is reflected in mental processes; new ideas are grasped with considerable effort that cannot be explained by reception of some processes.

If human brains were controlled receiving behavioral instructions from souls they would have to be even more complex than the brain/organism itself. Ability to survive would then depend on soul detailed knowledge of the physical world. The world of souls would have to contain the physical world to allow for learning. Imagine people living in the hunters/gatherers society who need to learn how to recognize, find and prepare for food hundreds of plants, seeds, roots, mushrooms. They must use all their senses and be aware of their actions to learn all skills needed for survival. It does not seem conceivable that such people make correct decisions guided by received conscious processes. Mental states are correlated with the brain states as can be seen in neuroimaging, and it is not possible to separate them, they are two sides of the same coin. If that interaction was local, like in the 
radio or TV receiver, where the signal from the antenna has to be amplified and used to create sounds and pictures, then some designated part of the brain would have to initiate the activity. However, there is no part of the brain where thinking or decision processes start, linear causality does not apply to network systems where collective states arise. The whole brain is engaged in semantic interpretation of sensory data, associations and memory recall. An alternative would be a two-way interaction with the whole cortex, large number of brain nuclei and some parts of the autonomous nervous system in the body. That would lead to a massive flow of information that would be hard to miss. Activity of each part of the brain is explained by the global interaction of local activations from other parts. There is no place for external driving forces here.

Brains working on solving hard problems use more glucose (as seen in PET imaging) and oxygen (as seen in fMRI signals). Receiver does not have to work harder to decide how to move a chess figure, but brains solving such problems have to do a lot of search, evaluating board positions and recalling memorized patterns that guide the search process. This is done largely at the unconscious level. Performing mental tasks that are well trained engages only a few brain areas, while performing novel tasks requires cooperation of the whole-brain network. A lot of research has shown that symbolic search and more recently pattern-based search in neural networks is a good model of brain processes that are responsible for thinking and problem solving. Two pioneers of artificial intelligence and cognitive sciences, Allen Newell and Herbert Simon, published in 1976 a paper "Computer Science as Empirical Inquiry: Symbols and Search”, that has defined physical symbolic systems as the basis for cognitive modeling. Ten years later this approach has been expanded to connectionist systems that use neural patterns as physical symbols (Rumelhart and McClelland, 1986).

\subsection{No function for souls}

In ancient times souls had precise functions: animation. Thomas Aquinas followed Aristotle postulating three kinds of souls for different types of 
movements. We have no problems understanding plant growth, explained by topobiology, and sensory-motor animal behavior. There is no more need for animation than in case of explosive chemical reactions.

If brain is a receiver of consciousness then what exactly is it receiving? Not the information that comes from senses or that is recalled from episodic memory. I am aware of sounds because my auditory cortex reacts to stimuli coming from the auditory nerves. Sounds are changed to neural impulses and decomposed into pitch, timbre, rhythm, or speech-specific reactions. There is nothing missing in this process, nothing for souls to do. I can recognize melodies, name the objects in front of me, because there is a flow of neural information to the recognition memory and a link with motor cortex storing phonological labels for brain activations. Processing steps from retina to inferior temporal cortex to speech areas are unraveled with growing number of details, and this knowledge is used as inspiration by computer vision systems.

Various forms of agnosia and anomia result from damages to the specific brain areas and processing pathways, and can be understood in terms of information processing by different brain areas. Visual and auditory illusions arise due to the extraction of specific information defining shapes, colors and movement. Activity of neurons in the primary visual cortex (V1) is only weakly correlated (about 10\%) with the signals from retina, saving brain processing power. Top down connections from the higher brain areas provide the missing information - to see and to have visual experiences one has to learn in the early childhood what is there to see. This is why millions of connections are formed at each second in the first few years of infant's life. Senses do not provide sufficient information for rich and detailed visual experiences; the expectations that have been learned are crucial. People who recover from blindness initially are not able to correlate their tactile experiences with what they see, but learn it within days or weeks. However, they experience simple illusions, such as Ponzo and Müller-Lyer, right after regaining sight (Gandhi et al. 2015). This is a result of specific way that visual cortex is analyzing the information and neural models explaining such illusions exist. The same image may lead to different conscious experiences 
(for example the Necker cube), depending on synchronization of groups of neurons in sensory cortices. Such illusions tell us a lot about consciousness (Dehaene 2014).

The sensory experience is a reflection of incoming stimuli in the neural substrate, it is a physical process. Qualia result from internal interpretation of sensory cortex activation. What then could be received that the brain is not able to produce by itself? Conscious perception takes place at the level that prepares us for action. We do not react to wavelength of reflected light but see red apples as red in a way that is largely independent of the type of illumination (color constancy effect). Visual cortex transforms the signal from retina to brain areas closer to motor cortex, simplifying the decision process. Some stimuli create only weak activity in the brain and are not distinguished from the neural noise, therefore we do not notice them consciously (this is explained in psychophysics by the signal detection theory, Swets 1966). Strong stimuli may be ignored if attention is focused on something else, because sensory signals do not propagate beyond primary sensory cortices (Dehaene 2003). Attention prepares the brain to receive specific information (Gilbert \& Sigman 2007), priming relevant cortices (neurons waiting for inputs are pre-activated, sending about 20 spikes per second), and the persistent signal reaches areas where it is accessible to motor and speech areas, allowing for verbal and non-verbal self-reflective conscious comments (Duch 2005).

Scholastic philosophers could not imagine computing machinery that we have now, and the principles that allow for information processing in neuromorphic systems. Understanding of reasoning or thinking mechanisms also does not require additional mechanisms. Thoughts and feelings seem to arise from nowhere, but using various neuroimaging techniques we can now observe neural processes that are correlated with subjective experiences. We can recreate sounds and sights from analysis of brain signals, and even say what people were dreaming of (Horikawa et al. 2013). The winner-takes-all neural mechanism leaves for a short while just one dominant process while others compete with each other until one of them wins and is propagated in the global neural workspace, becoming a part of the stream of consciousness. 
So what functions are left for the souls to perform? Willpower has been a subject of intensive research in recent years (Wegner 2002). It seems that the brain is preparing for various actions, and when preparation for selected motor activity becomes sufficiently strong other parts of the brain interpret it as the feeling of will. Our sense of agency is sometimes illusory. We may be acting but do not realize that we are - examples include ideomotor responses in ouija board movements used in spiritistic seances, facilitated communication, water divination and hypnotism. British neurologist William Carpenter already in 1852 described the muscular movement independent of conscious desires or emotions. The reverse situation is also possible: we may not be acting being convinced that we are. Subjects may be induced to believe that they have performed some actions, or that their actions are achieving far more than they in fact are (Wegner 2002). Conscious acts of will must arise from somewhere; otherwise there will be an infinite regress: to will to will to will ... Although we may be convinced that our decisions are free, initiated by conscious self, stimulation of the dorsolateral prefrontal cortex (DLPFC) with magnetic pulses or electric currents may significantly change them. The feeling of intention to act has been invoked by direct stimulation of the parietal cortex (Desmurget et al. 2009). Also neuropharmacological manipulation may have strong influence on decisions (for example, using oxytocin in spray). Brain stimulation may even induce immoral behavior (Glimcher \& Fehr 2003). The sense of agency and free will is generated by the brain as a result of external as well as intrinsic processes, not because of some signals received from souls. The only way to interpret our current knowledge about volitional processes is to assume that both conscious willing and action that follow or precede the feeling of will are the effects of a common unconscious brain processes (Hallet 2016).

\subsection{Animal minds}

The unity of nature is manifested at many levels, from molecules, proteins, genes, signaling pathways, biochemical cycles, body and brain structures, common to most animals. Reward, fear, arousal, affective, social and cogni- 
tive functions in animals are simplified versions of human brain functions. Big apes have much smaller brains than humans but show high intelligence, including planning, symbolic communication, emotions and feelings, a form of justice and culture, imagery and experience of insights (de Waal 1996, 2005), and even theory of mind (Krupenye et al. 2016). There is no doubt that animals are conscious of their environment and themselves. They possess minds, although with limited power of self-reflection. There is a clear correlation between brain complexity and powers of the intellect. Certainly in case of humans with much larger and complex brains new and more sophisticated functions should be expected. In particular development of language has enabled civilization, cooperation on a large scale, accumulation of knowledge, and gave some people enough time to create philosophy, science and high culture.

If human brain is a receiver then also animal brains should be receivers. A whole zoo of various souls would be needed. What kind of information they could receive? Animal brains are capable of generating quite sophisticated behavior. Many mammals (especially apes) need a long time to learn from their parents how to survive. They learn not only how to find food and shelter, but also learn social skills needed to collaborate with others. Their minds are created without the need for additional animation. Human brains create sophisticated minds that have greater intellectual powers due to the more complex brains, but this qualitative difference does not require completely new mechanisms that brains could not provide.

\subsection{Personal identity}

People are not conscious during deep sleep, in anesthesia or coma, and their ability to think and have mental experiences is impoverished in various disorders of consciousness and cognitive impairments. At the late stage of Alzheimer disease people lose the ability to recognize themselves and their family members, lose most of their intellectual powers. There is no rational soul left in them, although biological processes may still function for some time. Is it possible that their souls are conscious somewhere else? Does such concept make sense? 
If I do not know about my soul's conscious experience, how can this experience be called mine, be linked to my personal identity? Why should I care about it? Egyptians thought that everything that moves is alive. Shadow was considered to be a kind of independent entity, capable of independent life, because shadow moves. Should I care about my shadow? I am rather concerned about the neurodynamics of my brain, because any neurological damage may bring a lot of suffering. Although I cannot observe most processes that go on in my brain I know that if some of them stop working I will lose specific abilities, or will fall into coma. My personal identity is linked to my whole organism. I can identify with my brain and my body. I am conscious of using my fingers typing this sentence. Fingers are also a part of me at mental level, they allow me to function in a way I would not be able without them.

After heart transplantation more blood goes to the brain, neural networks function in a different way, and the stream of consciousness generated by brain activity becomes different. New thought associations may spontaneously arise, personal identity may be disrupted (Mauthner et al. 2015). If blood does not reach some parts of the brain, as it happens rather frequently in case of ischemic stroke, some functions are lost. Brain damage leads to specific impairments of cognition. Neuropsychology knows many cases of strange phenomena that change self-awareness, such as the feeling of phantom limbs, illusion of the third hand, autoscopic phenomena (illusions of leaving one's own body), various agnosias, including autopagnosia (body-image agnosia), or anosognosia or denial of existence of quite obvious disability (Morgan \& Ricker 2008). One brain may support a coherent network of various behaviors that define personality, and sometimes several networks may coexist, manifesting as multiple personalities, or alternative "selves". Million voices compete for conscious attention, trying to win control over global brain dynamics. Wrong brain wiring leads to abnormal behavior, for example distorted interpretation of pain signals (body dysmorphia, masochism), or pleasure from observed reactions to pain (sadism). A tumor in the brain may change normal people into pedophiles. Personality traits show significant variance around average values. Psychopaths are unable 
to feel empathy, and some people suffer from hyper-empathy, neglecting their own needs. Damage to prefrontal cortex frequently leads to acquired sociopathy, typical for affective/impulsive criminals. Damage to amygdala may lead to poor empathy, low fear, typical for psychopathic emotionless criminals. About $25 \%$ of all imprisoned in the USA belong to these two categories. Their ability for self-control is reduced because the reward system in their brain is not functioning properly. The field of moral neuroscience is rapidly growing (Liao 2016).

Such phenomena are explained by neural information processing in the brain and cannot be explained by reception of mind. This is simply what one should expect from a complex large brain dynamics. The damage to the receiver may only stop the reception, but will not change the content, create new associations or feelings. Neuropsychology and psychiatry shows that the content depends on brain processes and depends on local information processing. How can an illusion of the phantom limbs or the third hand be explained by reception errors? Or how can deep brain stimulation help in severe depression cases? Putative mechanisms responsible for such phenomena provide explanations that are now experimentally tested.

In psychology Self is understood as cognitive and affective representations of one's identity. Therefore Self has many aspects, it is not a unified entity. Experiments show that reaction to self-referential verbal, spatial, emotional and face recognition stimuli, when the task is to distinguish self from others, activates a whole network of cortical midline structures. These structures are placed deeper in the brain and therefore better protected from mechanical injuries. They link the surface of the cortex and limbic/ brain stem structures (Northoff et al. 2006).

Normal conscious experience requires activity that is both distributed over many local subnetworks, and integrated over most of the brain. It cannot be localized in one brain area. A complex dynamics of the whole neural system is involved in such processes. PET and fMRI studies show brain activity in normal awake subjects. Similar activity is seen in completely paralyzed locked-in subjects. Reduced neural activity is observed during anesthesia, disintegrated local activity persists in minimal consciousness 
states, and almost no activity is left in vegetative states of consciousness. Various mathematical measures are derived from brain signals to estimate the level of consciousness. Complexity of the structure is not sufficient by itself: cerebellum contains $80 \%$ of all neurons in the brain, but it's neural networks do not have sufficiently diverse structures to support various functions aspects of conscious information processing (selective attention, access to sensory and associative cortices implementing various forms of memory).

What can the concept of soul add to understanding of my personal identity? Suppose that some information about my Self could be preserved after the death of the body. Will using this information to restart conscious processes in a robot, or some resurrected body, or many identical copies of my brain and body, constitute me as the same person? Information by itself is not sufficient to create mind, but the same information embodied in identical physical system should duplicate all mental processes. Cloning the whole person should then create many identical selves, each feeling to be genuine but separate from others. They may think and feel like me but will not be me. This is a strange question, discussed in length by Hofstadter and Dennett (2001). Disembodied mind in some other world does not subsume my Self. Consciousness that is not connected to my Self cannot be mine.

\subsection{Artificial Intelligence and the mechanics of cognition}

Suppose that souls are indeed necessary to create intellect. Attempts to develop systems that think and perform similar functions as brains should then fail. Alan Turing has discussed this idea as one of possible objections against the possibility of building thinking machines, calling it a theological objection: machines have no souls, so they may not be able to think. He himself did not take this argument seriously, believing that computers should be able to pass intelligence test. What was only a belief in 1950 becomes reality in our times. Although logical approach to artificial intelligence, based on discrete symbols, failed to solve many AI problems hardware advances (speed and memory capacity) combined with new search and pattern-based algorithms gave spectacular results. In 1995 Chinook 
checkers program won 6:0 with world champion dr Tinsley, in 1997 Deep Blue won with Gary Kasparov in chess, and in 2011 IBM Watson won in Jeopardy with two best players in the history of this game. Although chess and checkers are board games with simple rules Jeopardy required very broad knowledge and some understanding of the questions asked. For a while it seemed that the strategic game of Go will be beyond machine reach for a long time, but in early 2016 Google AlphaGo won with the world champion Lee Sedol 4:1. This program is based on deep pattern analysis, not on the extensive search used by the computer chess programs. Recognition of faces and image analysis is already done in a better way by machines than humans. Soon driving vehicles of all sorts will also be left to computerized systems. The key to all this progress is machine learning.

Even quite simple parallel distributed processing systems, inspired by neural networks, have many properties characteristic of biological memory. Neural associative memories do not store information in localized places. Information is spread in connections between many units representing neurons. Distinct patterns of activity of these elements that persist for at least a short time represent memory traces, and transitions between them generate chains of thoughts. Memory in such models is retrieved from various cues (is content-addressable) and has associative character, linking related memory patterns. Errors that people make in memory tests, as well as their reaction times, may be replicated by associative memory models. Various forms of amnesia and memory related phenomena can be understood using neural modeling. Reward-based learning modifies network structure to reflect environmental events, creating internal model of objects and events encountered in the world. Computational models help to understand neuropsychological syndromes, psychiatric dysfunctions and cognitive aspects of neurological problems.

Computers are already better than brains in many applications that require thinking. In 2015 a laboratory based on robotic platform controlled by AI software discovered genetic and signal pathways responsible for regeneration of flatworm bodies, automatically forming hypothesis and performing experiments to verify them. Many attempts to create software 
for brain-inspired cognitive architectures (BICA) are under way, and robots controlled by such software are being built, from models of insects to serious NASA humanoid robots. Hector, insect robot that walks, plans its path, imagines alternative actions, has a number of higher-level mental states as a part of its control system (Cruse \& Schilling 2015). "Inner mental states" of Hector include intentions and goal-directed behavior guiding robot actions (find food = find charging station). Body properties are coupled with the environment and used in internal model for planning actions (second-order embodiment). Emotions are inherent properties of behavior implemented in the control model based on recurrent neural networks (RNN). "Depending on its inner mental state, the system may adopt quick, but risky solutions, [... or] take its time to search for a safer solution” (Cruse \& Schilling 2015).

Is there a natural limit to the powers of artificial intellect? Although existing solutions are in some respects far behind biological brains great progress in building hardware neuromorphic system has been made. The large consortium called "Synapse" led by IBM has announced in 2015 a new neuromorphic chip called TrueNorth. Each such chip contains over 5 billion transistors that implement one million artificial neurons and $250 \mathrm{mln}$ synapses (adjustable connections). 16 chips are combined in a module that has $16 \mathrm{mln}$ neurons with over 4 billion synapses, requiring only $2.5 \mathrm{Watt}$, very low power! Such systems may be scaled to the complexity comparable with the human brains. There is a chance that such hardware will allow for implementation of mental functions very similar to those of humans, although this has not yet been demonstrated.

There are no good arguments against convergence of the neural modeling process in the embodied robotic systems and brain-like structure to conscious artifacts (Duch 2005). Artificial minds of brain-like systems will have to claim qualia due to their construction. These qualia, comments on physically existing persistent internal dynamical states, may be as real in artificial systems as they are in our brains. NOMADs (neurally organized mobile adaptive device) brain-based devices are intentional robots endowed with simple instincts, learning from their own experience in simple environments, showing animal-like behavior (Edelman 2006). We may soon have 
more complex robots of this kind that will be aware of their environment, learning all skills needed for survival, including self-reflection. It should be possible to create artificial intellect that will surpass human abilities in most if not all respects. Advances in artificial intelligence, nanotechnology and neuromorphic chips point the way to construction of robots that will describe their inner states in mental terms. At some point we will have to acknowledge that they are conscious machines, even though their minds will differ from human minds. Essential features of being conscious, such as mental representation of the world, including construction of self, ability to comment on the flow of images in the brain, intellectual powers, are all within reach of technology. Psychopaths are conscious, therefore emotional reactions are not necessary to claim consciousness.

Artificial intelligence has not created yet conscious artefacts. Nevertheless, technology has reached the point in which construction of artificial systems based on neuromorphic chips may help to find empirical answers to questions about the nature of consciousness. It would be quite surprising to discover that certain functions of human mind could not be implemented in artificial intelligence systems because souls communicate only with biological brains.

\section{Matter, form and information}

Claiming that brain is a receiver of consciousness is equivalent to claiming that we have no idea how it works. This is simply ignorance, because we do know a lot about mechanics of brain processes, and know how to implement some of these mechanisms in artificial systems. The dualistic concept of soul is untenable for many reasons, some of which have been presented above. Connection of disembodied entities to material world has always been problematic. The hylomorphic understanding of mind in philosophy does not suffer from such problems. Mind may be understood as the specific form of highly organized matter that enables conscious processing of information. However, the concept of form has to be extended to account for dynamical nature of cognition. 


\subsection{Dynamical forms}

Matter is a form of energy, as we know from physics. Spatial form of matter carries information in its structure, giving matter specific properties. At the microscopic level spatial distances are very small and the time intervals are very short, everything is moving very fast. At larger scales changes are slow, therefore a kind of reification, symbolic description based on quantization of the phenomena, becomes possible. Time scale for changes of mental processes (perceptions, thoughts), and processes changing brain structure (learning, aging) allow for distinction between static and dynamical forms.

Form of the brain includes connectivity, neuronal structures, biochemistry, signaling pathways, genetics. The concept of form may be thus another word to describe structural phenotype that involves many levels of description. However, brains have more subtle dynamical form that rapidly changes in time and is the basis of mental states and behavioral functions. This dynamical form is observed in functional neuroimaging (fMRI), while anatomical form is observed using simpler structural imaging techniques. Computers may run infinite number of different programs that support quite different functionality. Brains that have the same physical form, structure of connections and properties of neurons, may support many functional, neurodynamical states. Each mental event - thought, feeling, intention changes this dynamical structure without changing the form of physical brain structures in a perceivable way. Of course at molecular level every cell changes in every moment, but at the macroscopic level these changes are negligible.

The patterns of electrical activity of neurons are the dynamic form of matter, carrying information. John Locke defined consciousness as "the perception of what passes in a man's own mind" (Locke 1975, II.i.19: 115). Some patterns are interpreted as perception of sensory stimuli and understood as external objects and events. Some patterns result from memory recall, and are understood as imagined. Some patterns are pointers to symbols, with phonological representation as motor commands that produce sounds. Yet another patterns are behavioral, motor commands leading to intentional actions. It all depends which brain structures are activated, how sensory, 
motor, associative memory and executive areas are activated. Patterns of brain activity are recognized by the brain itself, and associated with some form of imagery: visual, auditory (thoughts, words) or motor (actions).

Good examples of dynamical forms are found at the molecular level. The $\mathrm{C}_{25} \mathrm{H}_{52}$ molecule has 25 carbons and 52 hydrogens, but this matter may be arranged in about 36 million of different spatial forms, or isomers. Each spatial structure may be in many different dynamical states (electronic, vibrational and rotational excited states), most of which are stable only for a very short time. Each dynamical state has different properties, such as probability of interactions with other molecules or interactions with light, responsible for the absorption and emission spectra. Small molecules at quantum level may assume many discrete dynamical forms, influencing structural form to various degrees. For larger molecules or for highly excited states there are so many dynamical forms that differ in negligible way that in practice there is a continuum of possible forms.

Conscious processes are thus perception of dynamical forms, or synchronous activations, that arise in the brain. Scientists search for neural correlates of conscious processes (Koch et al. 2016), trying to characterize which patterns are perceived as conscious and which decay unnoticed. Learning processes change physical form, change brain connections and thus change the patterns that neurodynamics may activate. Studying such processes tells us which mental states (dynamical forms) are potentially accessible for the brains that have specific structure, depending on the individual connectome and other factors (neural properties, in particular ion channel types and their distribution, neurotransmitter release and many other factors).

While the current ideas in neuroscience can be linked with ancient hylomorphic understanding of the mind-body relations it will not help us to understand mental processes. Memory, personality traits and all mental faculties that can influence behavior are in a dormant, potentially accessible state until sufficient energy in specific form becomes available, facilitating their contribution at a given moment to the actual neurodynamics of the brain. Decay of the brain connections, or lack of specific forms of energy that activates neurons, destroys the system. Unless detailed information about 
the structure of the brain is stored this destruction is not reversible. Energy becomes dissipated in the form of heat, used in the decay processes, and information is lost. To prevent brain death after cardiac arrest temperature is decreased, and new resuscitation methods extend the time when the brain may be brought to life (currently this period is shorter than an hour). If the neural structure is not significantly damaged the brain may be "restarted" and the mind comes back. Freezing the whole body to $-80 \mathrm{C}$ or lower temperatures preserves the tissues. Cryoconservation techniques are not yet reversible, and it is not clear if hundreds of deeply frozen people will ever be able to come to life thanks to medical procedures.

\section{Conclusions}

Hylomorphism regards every physical object as a compound of matter and form. Form may be understood as specific organization of matter. Information defining structure is not material, information does not occupy space. Form in the brain has structural as well as dynamical aspects. While structural aspects define potentially accessible brain states dynamic aspects are responsible for actual, active states that are experienced as various mental phenomena. Dynamical form (neurodynamics) does not change brain structure; it defines a new level of description. At this level dynamical forms are identical to mental events. Techniques used in brain-computer interfaces, reading brain intentions, thoughts or imagery, are basically converting dynamical forms into sounds and images that other people may understand through normal sensory perception. Information is preserved, only representation is changed. From this point of view the gap between physical and mental processes is illusory.

Mental processes are supported by the brain that provides a substrate in which what is potentially possible may be actualized and become conscious experience. Dynamical form is an information process that changes the state of matter, but not the matter itself. Mind is thus truly non-materialistic, based on dynamical forms that exist only potentially, and is actualized by neurodynamics in a way that depends on many circumstances, including 
personal history. Self-observation of dynamical forms creates the spotlight of consciousness (Baars 1997).

Summarizing arguments presented above that the brain creates rather receives mind or conscious experience:

1. All functions proposed for souls have found natural explanations, so this concept has absent referent. Rational soul is equivalent to mind or intellect.

2. Mind is not monolithic, but a collection of many specialized functions. Mind received by the brain is not an intelligible concept.

3. Consciousness is not a thing that can be received, but a process that depends on the brain neurodynamics, parts of the brain commenting on states of other parts.

4. Detached minds would have to know all about this world to be able to transmit anything relevant to our activity and experience.

5. Even simple animal behavior is based on very complex physical processes; biological organisms are incredibly complex for a good reason.

6. Complex conscious experience must be supported by distinct patterns for different mental states, and that requires sufficiently complex substrate.

7. Brains are the most complex substrates in the known universe; such complexity is necessary for creation of mental states. Transmitter should be more complex than receiver.

8. Brains work hard solving problems; receivers should not require more energy.

9. Animal brains are similar to human and complexity of animal minds (social life, behavior, memory, problem solving) grows with the complexity of their brains, so their brains should also work as receivers.

10. Damages to the specific brain areas lead to strange neuropsychological syndromes, showing how experience is constructed by the brain. Auditory and visual illusions arise due to the specific construction of sensory cortices. 
11. Personal identification is based on memory and brain/body processes, it is also changing from infancy to the old age; one cannot identify with detached mind that is received.

12. The structure of connections and neural properties determines types of personality; it cannot depend on something received.

13. Some aspects of the neural processes responsible for perception, decision making and planning are already known and can be captured in artificial intelligence software.

Although I have focused here on arguments showing how brains create minds it should be remembered that only a coarse structure of the brain is created by biological factors (genetic, epigenetic, developmental processes). A small number of neural connections in the brain of newborns develops in the span of a few years through interactions with the external world into a million billion connections, internalizing the knowledge about the world, enabling perception and thinking. Brains can change themselves even in the older age (Doidge 2007) due to neuroplasticity. What we see, hear, experience, think changes brain structure all the time. Psychology describes limitations of mental processes that brains of particular type are able to carry. Research on psychotherapy and neuroplasticity explores the ways that mental processes influence brain structures (Cozolino 2010). Therefore it may also be said that minds create brains through top-down causality. This position has been central to non-reductive physicalism of Nancy Murphy (2006), who has also asked what is left for a soul to do? The concept of a dynamical form presented here is indeed an emergent phenomenon. Although it needs material substrate it cannot be reduced to physical reality.

\section{References}

Aquinas, St. Thomas. 1920. The Summa Theologice, $2^{\text {nd }}$ Revised Edition, Translated by Fathers of the English Dominican Province.

Aristotle: De Anima. 2016. Oxford, New York: Oxford University Press. 
Baars, B. 1997. In the Theater of Consciousness: The Workspace of the Mind, NY: Oxford University Press.

Bassett, D.S., \& Gazzaniga, M.S. 2011. "Understanding complexity in the human brain." Trends in Cognitive Sciences 15(5):200-209.

Bering, J.M. 2006. "The folk psychology of souls." Behavioral and Brain Sciences 29:453-498.

Beauregard, M. \& O'Leary D. 2007. The Spiritual Brain: A Neuroscientist's Case for the Existence of the Soul, Harper Collins.

Cleeremans, A. 2011. "The radical plasticity thesis: how the brain learns to be conscious." Frontiers in Psychology 2:59-70

Cozolino, L. 2010. The Neuroscience of Psychotherapy: Healing the Social Brain. New York: W. W. Norton \& Company.

Cruse H. \& Schilling M. 2015. "Mental States as Emergent Properties. From Walking to Consciousness." In T. Metzinger, ed. Open MIND Project.

Dehaene, S. 2014. Consciousness and the Brain: Deciphering How the Brain Codes Our Thoughts. Penguin Books.

Dehaene, S., Sergent, C. \& Changeux, J.-P. 2003. “A neuronal network model linking subjective reports and objective physiological data during conscious perception.” PNAS 100(14):8520-8525.

Desmurget, M., Reilly, K.T., Richard, N., Szathmari, A., Mottolese, C., Sirigu, A. 2009. “Movement Intention After Parietal Cortex Stimulation in Humans." Science 324:811-813.

Doidge, N. 2007. The Brain That Changes Itself: Stories of Personal Triumph from the Frontiers of Brain Science. New York: Penguin Books.

Duch, W. 2005. “Brain-inspired conscious computing architecture.” Journal of Mind and Behavior 26(1-2):1-22.

Duch W. 2012. "Mind-Brain Relations, Geometric Perspective and Neurophenomenology.” American Philosophical Association Newsletter 12(1): 1-7

Eccles, J.C. \& Popper, K. 1991. Evolution of the Brain: Creation of the Self. New York: Routledge.

Edelman, G. 2006. Second Nature: Brain Science and Human Knowledge. Yale University Press.

Edelman, G.M. 2007. “Learning in and from Brain-Based Devices.” Science 318:1103-1105 .

Gandhi, T., Amy Kalia, A., Ganesh, S. \& Sinha, P. 2015. „Immediate susceptibility to visual illusions after sight onset.” Current Biology 25(9), R358-R359.

Gilbert, C. \& Sigman, M. 2007. "Brain States: Top-Down Influences in Sensory Processing.” Neuron 54(5), 677-696. 
Glimcher, P.W. \& Fehr E. (eds) 2013. Neuroeconomics: Decision Making and the Brain. 2nd ed, Elsevier.

Hallett, M. 2016. "Physiology of free will.” Annals of Neurology 80(1): 5-12.

Hofstadter, D. R., \& Dennett, D.C. 2001. The Mind's I: Fantasies And Reflections On Self \& Soul. New York: Basic Books.

Horikawa, T., Tamaki, M., Miyawaki, Y., \& Kamitani, Y. 2013. "Neural Decoding of Visual Imagery During Sleep”. Science, 340:639-42.

Koch, C., Massimini, M., Boly, M., \& Tononi, G. 2016. „Neural correlates of consciousness: progress and problems.” Nature Reviews Neuroscience 17:307-321.

Krupenye, C., Fumihiro Kano, F., Hirata, S., Call, J. \& Tomasello. M. 2016. "Great apes anticipate that other individuals will act according to false beliefs." Science 354(6308):110-114.

Laureys, S., Owen, A.M., \& Schiff, N.D. 2004. "Brain function in coma, vegetative state, and related disorders." Lancet Neurology 3:537-546.

Liao, S.M. 2016. Moral Brains: The Neuroscience of Morality. Oxford University Press. Locke, J. 1975. An Essay Concerning Human Understanding. Ed. Peter H. Nidditch. Oxford: Clarendon Press.

Mauthner, O.E., De Luca, E., Poole, J.M., Abbey, S.E., Shildrick, M., Gewarges, M., \& Ross, H.J. 2015. "Heart transplants: Identity disruption, bodily integrity and interconnectedness." Health: An Interdisciplinary Journal for the Social Study of Health, Illness and Medicine 19(6):578-594.

Minsky, M. 1986. The Society of Mind. New York: Simon \& Schuster.

Morgan J.E. \& Ricker J.H. (Eds). 2008. Textbook of Clinical Neuropsychology. Taylor $\&$ Francis.

Murphy, N. 2006. Bodies and Souls, or Spirited Bodies? Cambridge University Press.

Newell, A. \& Simon, H. 1976. “Computer Science as Empirical Inquiry: Symbols and Search.” ACM Journal 19:113-126.

Northoff, G., Heinzel, A., de Greck, M., Bermpohl, F., Dobrowolny, H. \& Panksepp, J. 2006. "Self-referential processing in our brain, a meta-analysis of imaging studies on the self." Neuroimage 31:440-457.

Parnia, S. 2014. "Death and consciousness - an overview of the mental and cognitive experience of death." Ann. New York Academy of Science 1330(1):75-93.

Rumelhart, D.E., McClelland, J.L. \& PDP Research Group. 1986. Parallel Distributed Processing: Explorations in the Microstructure of Cognition. Cambridge, MA: MIT Press.

Schwitzgabel, E. 2011. Perplexities of Consciousness. MIT Press. 
Sleutjes, A., Moreira-Almeida, A., \& Greyson, B. (2014). "Almost 40 Years Investigating Near-Death Experiences: An Overview of Mainstream Scientific Journals.” The Journal of nervous and mental disease, 202(11):833-836.

Swets, J.A. (ed.) 1964. Signal detection and recognition by human observers. New York: Wiley

de Waal, F. 1996. Good Natured: The Origins of Right and Wrong in Humans and Other Animals. Cambridge, MA: Harvard University Press.

de Waal, F. 2005. Our Inner Ape. Granta Books, London.

Wegner, D.M. 2002. The illusion of conscious will. MIT Press. 